\title{
Can party balloons replace autoinflation balloons to treat glue ear? A technical comparison
}

\author{
Katherine Marshall1, Rafael Perera², Paul Glasziou ${ }^{3}$, Susannah Fleming ${ }^{2 *}$ \\ ${ }^{1}$ Medical School, University of Oxford, Oxford, UK; ${ }^{2}$ Nuffield Department of Primary \\ Care Health Sciences, University of Oxford, Oxford, UK; ${ }^{3}$ Faculty of Health Sciences \\ \& Medicine, Bond University, Robina, QLD, Australia
}

\begin{abstract}
Background: Autoinflation balloons are used to treat patients with otitis media with effusion (OME) to help avoid surgery.

Aim: To compare the ability of party balloons with Otovent balloons to produce sufficient pressure for a Valsalva manoeuvre.

Design \& setting: Pressure testing was used to determine the number of times each balloon could produce pressures sufficient for a Valsalva manoeuvre. Subsequently, Otovent balloons were compared with spherical party balloons in a pilot clinical trial of 12 healthy adults.
\end{abstract}

Method: Each balloon was inflated 20 times and the maximum pressure was recorded. Three balloons of each type were tested to 50 inflations to assess pressures over persistent use.

Results: Otovent balloons' mean inflation pressure was $93 \mathrm{mmHg}(95 \%$ confidence interval $[\mathrm{Cl}]=89$ to $97 \mathrm{mmHg})$ on first inflation, dropping to $83 \mathrm{mmHg}(95 \% \mathrm{Cl}=80$ to $86 \mathrm{~mm} \mathrm{Hg})$ after 20 inflations. Two types of spherical party balloon required mean inflation pressures of $84 \mathrm{mmHg}(95 \% \mathrm{Cl}=77$ to $90 \mathrm{mmHg})$ and $108 \mathrm{mmHg}(95 \% \mathrm{Cl}=97$ to $119 \mathrm{mmHg})$ on first inflation, dropping to $74 \mathrm{mmHg}(95 \%$ $\mathrm{Cl}=68$ to $81 \mathrm{mmHg})$ and $83 \mathrm{mmHg}(95 \% \mathrm{Cl}=77$ to $88 \mathrm{mmHg})$ after 20 inflations. In the pilot trial, there was no difference between the ability of Otovent and spherical balloons $\left(\chi^{2}=0.24, P=0.89\right)$ to produce the sensation of a Valsalva manoeuvre.

Conclusion: Otovent balloons can be used more than the 20 times quoted by the manufacturer. The two spherical balloons produced similar pressures to Otovent balloons, indicating potentially the

*For correspondence: susannah. fleming@phc.ox.ac.uk

Competing interest: See page 7

Received: 20 November 2020

Accepted: 19 January 2021

Published: 21 April 2021

(C)This article is Open Access: CC BY license (https://creativecommons.org/licenses/by/4.0/)

Author Keywords: otitis media with effusion, Valsalva maneuver, pediatrics, primary health care, general practice

Copyright (C) 2021, The Authors; DOI:10.3399/BJGPO.2020.0178 same clinical effect. The pilot study suggests a potential use of spherical party balloons instead of Otovent balloons as a cost-efficient treatment.

\section{How this fits in}

Autoinflation balloons, such as Otovent, can be used as an effective treatment option in OME. This research shows that two types of spherical party balloons are able to produce similar pressures to Otovent balloons. It also shows that both Otovent and spherical party balloons are capable of producing sufficient pressure for a Valsalva manoeuvre for at least 50 inflations. This provides the potential for reduced cost autoinflation treatment for children with OME.

\section{Introduction}

OME, commonly known as glue ear, is a non-inflammatory fluid blockage in the middle ear, ${ }^{1}$ without symptoms of acute infection. Grommet insertion to treat glue ear is the most common reason for a surgical procedure in childhood in the UK, with 25000 procedures performed each year. ${ }^{2}$ OME has 
a point prevalence of $20 \%$ in 2 year olds, ${ }^{3}$ and by the age of 4 years has a cumulative incidence of $80 \% .{ }^{4}$ OME can lead to conductive hearing loss; for patients with bilateral OME for over a month, this can be as great as $20-30 \mathrm{~dB}^{5}{ }^{5}$ This hearing loss resolves in $95 \%$ of cases within a year, ${ }^{6}$ although patients may experience linguistic, developmental, and behavioural consequences. ${ }^{7}$ Current National Institute for Health and Care Excellence (NICE) guidelines suggest a 3-month period of watchful waiting $^{8}$ as OME has been shown to resolve in $50 \%$ of cases during this period. ${ }^{9}$ Furthermore, in children aged $<3$ years, a delay of 6 months in surgical treatment does not appear to significantly affect developmental outcomes. ${ }^{10}$ Medical treatment options include mucolytics, antihistamines, corticosteroids, decongestants, ${ }^{11}$ and antibiotics. ${ }^{12}$ Antibiotic treatments show either short-lived benefit for 1 month ${ }^{13}$ or no benefit at all. ${ }^{14}$ Corticosteroids can be effective in the short term, ${ }^{15}$ although no long-term benefit has been shown. ${ }^{16}$

Autoinflation balloons can be used to treat $\mathrm{OME}$, as shown in a recent systematic review, ${ }^{17}$ with a number needed to treat of $9 .{ }^{18}$ These balloons equalise the pressure between the middle ear and nasal cavity. The intranasal pressure is increased on inflation of the balloon, forcing open the eustachian tube and equalising the negative pressure. This process is a Valsalva manoeuvre, ${ }^{19}$ with 40 $\mathrm{mmHg}$ usually quoted for an effective Valsalva manoeuvre (range of $30-50 \mathrm{mmHg}$ ). ${ }^{20-24}$ The balloon provides a visual aid to help the patient complete a Valsalva manoeuvre. Otovent balloons are a type of autoinflation balloon sold as a set of five with a nosepiece. The balloon is fitted to the flat end of the nosepiece while the ball-shaped section is placed to form a tight seal against the nostril; the other nostril is compressed. ${ }^{25}$ Manfacturers of Otovent balloons recommend a maximum of 20 inflations per balloon, so Otovent balloons were tested to determine after how many repeated inflations they could produce a pressure of $40 \mathrm{mmHg}$. Four different types of party balloons were also tested to determine whether they could reach adequate pressures to be used as a potential alternative method for the treatment of OME.

\section{Method}

A novel protocol was designed using the Omron PA350e blood pressure monitor tester to inflate the balloons and measure the pressure (Figure 1a). The pressure-testing device has previously been used to assess the accuracy of sphygmomanometers used in primary care. ${ }^{26}$ This apparatus consisted of three pieces of rubber tubing linked together by connectors, with one end attached to the nosepiece designed for Otovent balloons. ${ }^{25}$ To inflate the balloon, air was pumped through the plastic tubing into the balloon allowing the pressure within to be measured.

As the balloon inflated, the pressure within rose until a critical point when the volume of air within the balloon continued to increase but the pressure decreased. After the maximum pressure had been reached by each balloon (shown by a decline in pressure despite an increase in volume) the machine was turned off, allowing the balloon to deflate. This process was repeated 20 times unless the balloon
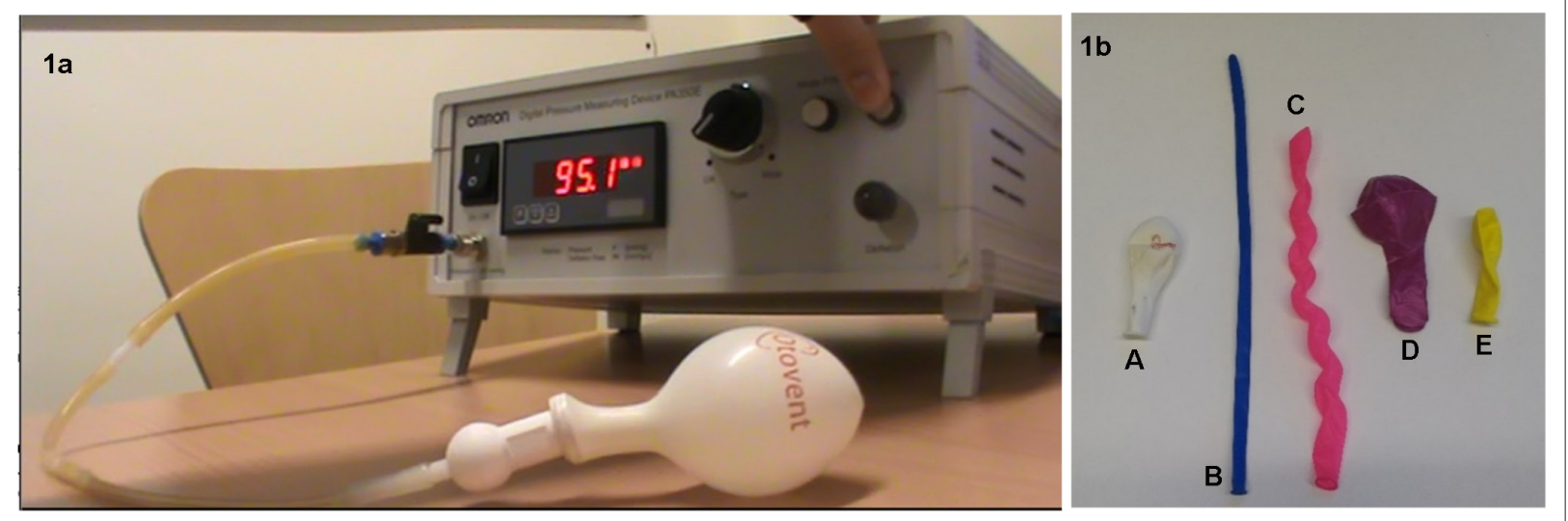

Figure 1 Test equipment used in the study. (Figure 1a) Omron PA350e blood pressure monitor used to test the maximum pressure achieved by each balloon, connecting tubes, nosepiece, and Otovent balloon. (Figure 1b) Balloons tested, left to right: Otovent (A), modelling (B), spiral (C), sphere 1 (D), sphere $2(E)$ 
burst, as this is the limit quoted by Otovent. Each inflation was video-recorded, enabling the digital display of the machine to be checked to determine the maximum pressure reached by each balloon.

Five different types of balloons were tested (Figure 1b); eight Otovent balloons (A), and 10 party balloons of each of four different types (modelling [B], spiral [C], sphere $1[D]$, and sphere 2 [E]). The party balloons selected were easily available and included a range of shapes, sizes, and manufacturers. The modelling balloons were long and thin, while the spiral balloons were long and irregularly shaped. The Otovent (A) and sphere 2 (E) balloons were small and spherical; sphere 1 (D) balloons were larger in size but had the same shape. All available colours of balloons were tested to give an initial indication as to whether colour affected the maximum pressure of the balloon.

The spiral balloons, owing to their irregular shape, required a maximum pressure to be recorded after 5 seconds as well as after 30 seconds. This allowed the pressures within the balloon to be acurrately determined throughout the course of the 20 inflations. Four of the 10 modelling balloons did not inflate despite being pressurised to $300 \mathrm{mmHg}$ (the maximum pressure produced by the testing device) and, therefore, were removed from the analysis.

In addition, three of each of the Otovent (A), sphere $1(D)$, and sphere 2 (E) balloons were subjected to an extended test of 50 inflations to determine if they were still able to achieve the pressure of 40 $\mathrm{mmHg}$.

The data for each inflation number for each balloon was summarised by mean and Cls. Linear regression was then used to analyse the data. These equations were used to extrapolate the data until the point at which a Valsalva manoeuvre could no longer occur (maximum pressure of $40 \mathrm{mmHg}$ ). Following this, a linear mixed-effects model was used with both varying intercept and varying slope, allowing for the original grouping of the data by individual balloon. This model allowed variability between different balloons of the same type to be determined. Furthermore, this data was used to determine the longevity of the 'worst predictable' balloon. This was determined by finding the mean minus 2SD for the intercept and slope. All Cls are $95 \%$, and results were considered statistically significant if the $P$ value was $<0.05$. Analyses were performed in $R$ (version 3.3.1).

\section{Pilot clinical trial}

Following the successful tests of both spherical balloons ( $D$ and $E$ ), a pilot clinical trial was carried out to determine their effectiveness in ability to perform a Valsalva manoeuvre in comparison with Otovent balloons (A). Participants were healthy, aged 18-65 years and recruited via word of mouth. Exclusion criteria included having history of recent nasal trauma or bruising; severe coronary artery disease; a moderate to severe reduction in blood volume; or having experienced a recent heart attack. Informed consent was achieved by a verbal explanation of the study at the time of consent as well as a participant information sheet; 12 participants gave their written informed consent.

Participants were randomised into three groups and used the balloons in three different orders (ADE, DEA, and EAD, respectively). It was not possible to use a placebo or to double-blind the trial by masking which balloons were being used. Each participant was asked to inflate the three balloons in turn by placing the balloon to one nostril, covering the other, and breathing out into the balloon; it was recorded whether they felt their ear 'pop'. Inflation was carried out without a nosepiece for

Table 1 Mean and 95\% confidence intervals of maximum pressures ( $\mathrm{mmHg}$ ) achieved during the first 30 seconds of inflation for each balloon type

\begin{tabular}{|c|c|c|c|c|c|c|c|}
\hline \multirow[b]{2}{*}{ Balloon type } & \multicolumn{3}{|c|}{ Initial inflation } & \multicolumn{2}{|c|}{ After 20 inflations } & \multicolumn{2}{|c|}{ After 50 inflations } \\
\hline & $n$ & Mean $(\mathrm{mmHg})$ & $\begin{array}{l}\text { 95\% confidence interval } \\
(\mathrm{mmHg})\end{array}$ & $\begin{array}{c}\text { Mean } \\
(\mathrm{mmHg})\end{array}$ & $\begin{array}{l}\text { 95\% confidence interval } \\
(\mathrm{mmHg})\end{array}$ & Mean $(\mathrm{mmHg})$ & $\begin{array}{l}95 \% \text { confidence interval } \\
(\mathrm{mmHg})\end{array}$ \\
\hline Otovent (A) & 8 & 92.9 & 88.9 to 96.8 & 82.7 & 79.6 to 85.7 & 77.4 & 74.0 to 80.8 \\
\hline Modelling (B) & 10 & 278.1 & 221.14 to 335.0 & 107.5 & 75.7 to 139.2 & $\mathrm{n} / \mathrm{a}$ & $\mathrm{n} / \mathrm{a}$ \\
\hline Spiral -5 seconds (C) & 10 & 79.77 & 70.6 to 89.0 & 58.5 & 53.6 to 63.4 & $\mathrm{n} / \mathrm{a}$ & $\mathrm{n} / \mathrm{a}$ \\
\hline Spiral - 30 seconds (C) & 10 & 74.77 & 64.6 to 84.8 & 62.1 & 57.5 to 66.7 & $\mathrm{n} / \mathrm{a}$ & $\mathrm{n} / \mathrm{a}$ \\
\hline Sphere 1 (D) & 10 & 83.6 & 77.3 to 90.0 & 74.0 & 67.5 to 80.5 & 71.2 & 66.5 to 75.9 \\
\hline Sphere $2(E)$ & 10 & 107.7 & 96.5 to 118.9 & 82.6 & 77.3 to 87.9 & 77.1 & 67.8 to 86.4 \\
\hline
\end{tabular}




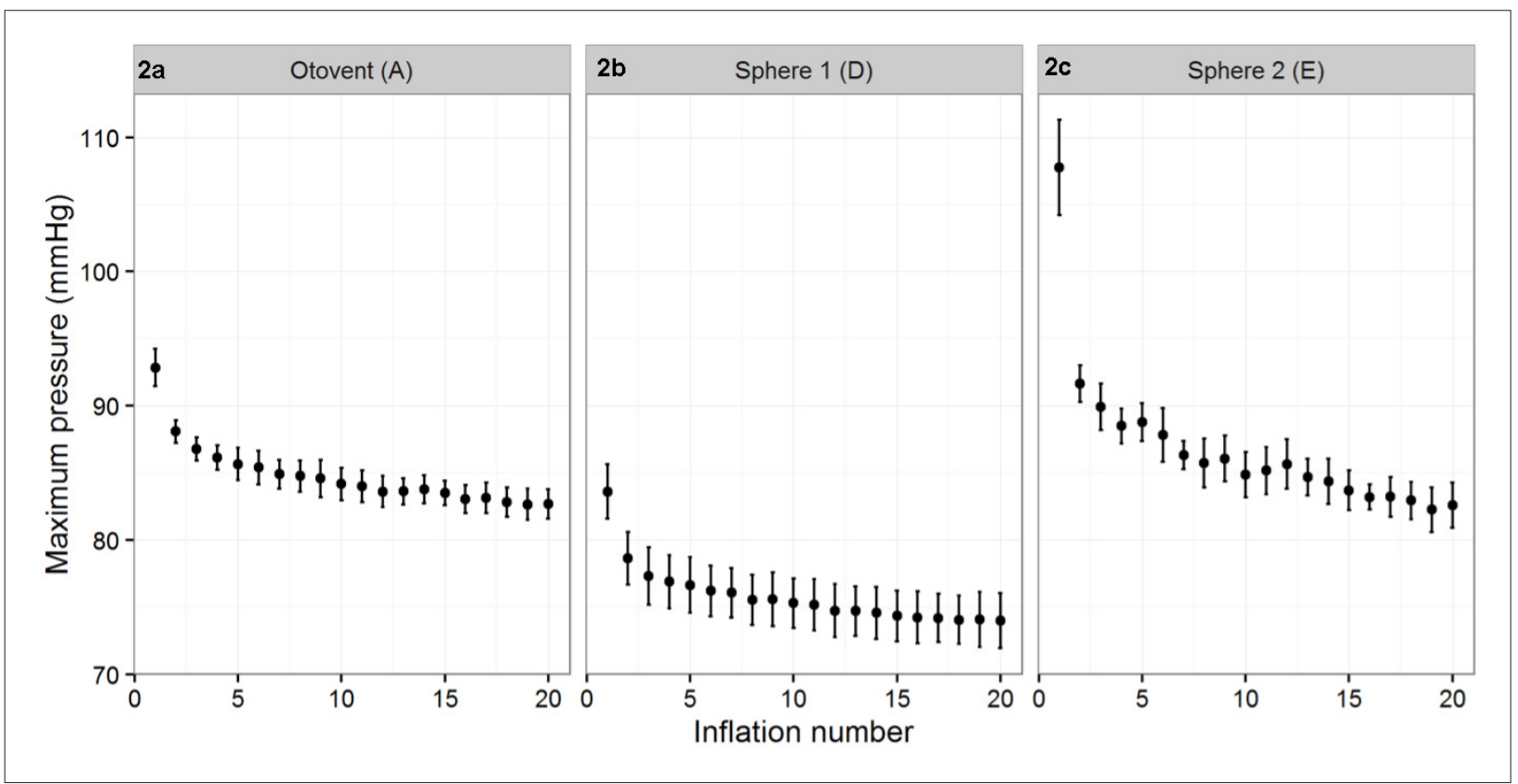

Figure 2 Mean inflation pressures (2-20) and standard deviation of (Figure 2a) Otovent (A), $n=8$; (Figure 2b) sphere 1 (D), $n=10 ;($ Figure 2c) sphere 2 $(\mathrm{E}), n=10$.

all balloons. If this occurred, a Valsalva manoeuvre was deemed to have successfully taken place. A successful Valsalva manoeuvre was recorded as either a 'yes' or 'no' to ear popping. The results were analysed by finding the percentage success rate and followed by a $\chi^{2}$ test of independence to compare the groups.

\section{Results}

Table 1 shows the mean inflation pressures and $\mathrm{Cls}$ for all balloons at initial inflation; after 20 inflations (Figure 2, supplementary Figures S2 and S3); and, where relevant, after 50 inflations (Figure S1). The modelling balloons (B) were found to require excessively high inflation pressures. The long spiral balloons $(C)$ had an unpredictable and variable pressure response during inflation, with two balloons bursting, making their results unreliable. The Otovent balloons $(A)$ achieved the required pressure throughout testing, along with both types of spherical party balloon ( $D$ and $E$ ). All of these balloons showed linear decreases in the pressure required to inflate them from the second inflations.

\section{Linear regression analysis}

Sphere 1 (D) had the shallowest gradient closely followed by Otovent (A) and then sphere 2 (E). Sphere 2 (E) had the highest intercept, before Otovent (A) and then sphere 1 (D) (supplementary Figure S4). The results were extrapolated to predict how many times the balloons could be inflated before they were no longer able to carry out a Valsalva manoeuvre (Table 2).

Table 2 Linear regression results (inflation number 2-50) for Otovent (A), sphere 1 (D), and sphere 2 (E)

\begin{tabular}{|c|c|c|c|c|c|c|}
\hline Balloon & $n$ & Equation & $\mathrm{Cl}$ intercept & $\mathrm{Cl}$ gradient & $P$ value of gradient & $\begin{array}{l}\text { Predicted number of inflations } \\
\text { when maximum pressure is } 40 \mathrm{mmHg}\end{array}$ \\
\hline Otovent (A) & 8 & $Y=-0.1775^{\star} X+86.63$ & $\begin{array}{l}86.23 \text { to } \\
87.02\end{array}$ & $\begin{array}{l}-0.191 \text { to } \\
-0.1641\end{array}$ & $<0.0001$ & 262 \\
\hline Sphere 1 (D) & 10 & $Y=-0.1108 * X+77.13$ & $\begin{array}{l}76.49 \text { to } \\
77.77\end{array}$ & $\begin{array}{l}-0.1323 \text { to } \\
-0.0893\end{array}$ & $<0.0001$ & 335 \\
\hline Sphere $2(E)$ & 10 & $Y=-0.2355^{\star} X+88.70$ & $\begin{array}{l}88.05 \text { to } \\
89.34\end{array}$ & $\begin{array}{l}-0.2574 \text { to } \\
-0.2136\end{array}$ & $<0.0001$ & 206 \\
\hline
\end{tabular}


Table 3 Mixed-effect model for inflation numbers 2-50: results and inflation number of 'worst predictable' balloon for Otovent (A), sphere $1(D)$, and sphere 2 (E).

\begin{tabular}{|c|c|c|c|c|c|c|}
\hline \multirow[b]{2}{*}{ Balloons } & \multirow[b]{2}{*}{$n$} & \multicolumn{2}{|c|}{ Intercept } & \multicolumn{2}{|c|}{ Slopes } & \multirow{2}{*}{$\begin{array}{l}\text { 'Worst predictable balloon' } \\
\text { inflation number when maximum } \\
\text { pressure is } 40 \mathrm{mmHg}\end{array}$} \\
\hline & & Mean & SD & Mean & SD & \\
\hline Otovent (A) & 8 & 86.75 & 1.446 & -0.204 & 0.0163 & 185 \\
\hline Sphere 1 (D) & 10 & 77.50 & 3.384 & -0.18 & 0.0866 & 87 \\
\hline Sphere 2 (E) & 10 & 90.20 & 2.377 & -0.402 & 0.156 & 63 \\
\hline
\end{tabular}

\section{Mixed-effect model}

To analyse the variability between different balloons of the same type, a mixed-effect model was used. Sphere 2 (E) had the highest intercept followed by Otovent balloons (A) and sphere 1 (D), as shown in Table 3. Sphere 1 (D) had the shallowest gradient, followed by Otovent (A) and then sphere 2 (E). When comparing the 'worst predictable balloon' model, Otovent (A) balloons showed the least variability and had the highest number of inflations before being unable to complete a Valsalva manoeuvre (maximum pressure dropped below $40 \mathrm{mmHg}$ ). Sphere 1 (D) balloons lasted for more inflations than sphere 2 (E) balloons, but both had a lower maximum inflation number than the Otovent (A) balloon. A 'worst predictable balloon' model was calculated using mean-2SD for both the intercept and slope.

\section{Pilot clinical study results}

Participants had an equal success rate with Otovent balloons (A) and sphere 2 (E) balloons (Table 4). The $\chi^{2}$ test showed no difference between the three balloon groups $\left(\chi^{2}=0.2408, P=0.887\right)$.

\section{Discussion}

\section{Summary}

Overall, it has been shown that Otovent balloons can be used more than the 20 times printed on the manufacturer's guidelines, and will still be able to produce a Valsalva manoeuvre after at least 50 uses. Furthermore, it has been shown that spherical party balloons are able to produce similar pressures for a similar number of inflations. Balloon shape is an important factor in the use of party balloons, as neither the modelling nor spiral balloons were effective owing to the long and either irregular or thin shape. As the current NICE guidelines ${ }^{8}$ suggest a period of watchful waiting for the first 3 months before any surgical treatment, the use of spherical party balloons could provide a cost-efficient treatment option during this time period. Extrapolating the linear regression and mixed-model data suggests that it would take over nine times the manufacturer's limit of 20 inflations before Otovent balloons were no longer able to reach the necessary pressure. However, party balloons (sphere 1 [D] and sphere 2 [E]) are still able to be inflated at least four times and three times respectively more than the Otovent manufacturer's limit (even when looking at the 'worst predictable balloon' extrapolation), showing their possible effectiveness in clinical practice. Autoinflation balloons were able to be tested against spherical balloons for the first time in healthy patients allowing their effectiveness at performing a Valsalva manoeuvre and their subsequent

Table 4 Success rates of Otovent (A), sphere 1 (D), and sphere 2 (E) in causing a Valsalva manoeuvre when inflated $(n=12)$

\begin{tabular}{lccc}
\hline & \multicolumn{3}{c}{ Success rate } \\
\cline { 2 - 4 } Balloons & $\mathbf{n}$ & $\begin{array}{c}\text { Mean } \\
(\%)\end{array}$ & $\begin{array}{c}95 \% \\
\text { confidence } \\
\text { interval (\%) }\end{array}$ \\
\hline Otovent (A) & 12 & 66.7 & 40.0 to 93.3 \\
\hline Sphere 1 (D) & 12 & 58.3 & 30.4 to 86.2 \\
\hline Sphere 2 (E) & 12 & 66.7 & 40.0 to 93.3 \\
\hline
\end{tabular}
potential use in the treatment of OME.

\section{Strengths and limitations}

A range of party balloons were able to be tested from a variety of different manufacturers. Guidance for the use of Otovent balloons suggests that an adult inflates the balloon for the first time to show the child how to use it and to overcome the larger pressure required for the first inflation. This further supports the use of sphere party balloons as the larger pressure 
required to initially inflate sphere 2 (E) balloons (Table 3 ) could be negated by adult assistance. All balloons were tested under repeated stress that was greater than would have been experienced by normal use. Inflations for each balloon happened in quick succession compared with three inflations per day in ordinary use of Otovent balloons. This was demonstrated by a pressure increase between inflations 20 and 21 (supplementary Figure S1), as two of the three balloon types did not have the final 30 inflations carried out immediately after the first 20. This might suggest that the balloons could still achieve the required pressures for even more inflations if the intervals between inflations were longer.

The analysis considers the variability between different balloons tested, although, as the values are extrapolated, an individual balloon may burst before this point. The main limitation was the use of the nosepiece to attach all balloons to the tubing connected to the pressure monitor. If spherical party balloons in treatment were used as opposed to Otovent, then the nosepiece would not be available; this could prevent a seal being created to inflate the balloons. However, in the pilot clinical trial, participants still reported a Valsalva manoeuvre despite the absence of a nosepiece. It was not possible to blind the participants as to which balloon they were using, although this reflected how these balloons would be used in routine practice. The sample size of the pilot clinical trial was limited by the number of Otovent balloons remaining from the original data collection and, consequently, so was the power of the analysis. Therefore, it would only have been possible to detect large differences between the three groups.

The experiment did not assess the effect of different inflation speeds, or of different volumes required to attain a pressure sufficient to achieve a Valsalva manoeuvre. The equipment used inflates at a roughly constant speed, which is probably faster than that which could be achieved by a person, particularly a child.

\section{Comparison with existing literature}

In comparison with a previous study with party balloons, ${ }^{27}$ the ability of specialised autoinflation balloons was compared with party balloons. Also, unlike previous experiments, the maximum pressure reached by each balloon via a pressure monitor was determined in order to assess whether a Valsalva manoeuvre could take place. Recent randomised clinical trials ${ }^{28}$ suggest that autoinflation is an effective way for treating OME during the watchful-waiting period and this study's results confirm the reliability of Otovent balloons to consistently reach the required pressure for a Valsalva manoeuvre.

\section{Implications for research and practice}

The results have suggested that spherical party balloons and Otovent balloons have limited differences for patients owing to the 'all-or-nothing' nature of a Valsalva manoeuvre. This implies that spherical party balloons could be used instead of Otovent balloons for treatment of OME, which would provide large cost savings. Otovent balloons are sold in a pack of five and, at drug tariff prices, are $£ 0.98$ per balloon, while the spherical balloons used in this study are sold in packs of 50 and average $£ 0.029$ per balloon. The recommended treatment time for autoinflation balloons is between 1 and 3 months (during the NICE guideline recommended watchful-waiting period). Therefore, if a balloon has a maximum of 20 inflations (based on Otovent instructions) and is to be used three times per day, five balloons would be required per month of treatment. For 1 month, the cost would be $f 0.15$ (spherical) compared with $£ 4.90$ (Otovent), while for 3 months of treatment this would be $f 0.45$ (spherical) compared with $£ 14.70$ (Otovent). Further savings could be obtained if a larger number of inflations were used before discarding a balloon, which the data indicates may be possible.

Further experiments need to be carried out in a randomised control trial to compare the use of spherical party balloons with specialised Otovent balloons on a paediatric patient group with OME, to test compliance as well as effectiveness. Otovent balloons have been shown to have a compliance rate of $80 \%$ at 3 months. ${ }^{18}$ It would also be important to test whether or not a nosepiece is necessary to create the seal required to reach the necessary pressure to perform a Valsalva manoeuvre, especially in a young child under supervision. An alternative could be to use a nosepiece initially and then transition to using party balloons with or without the original nosepiece. Further research is also needed to test whether there is an infection risk of repeatedly using any balloon type beyond the 20 inflation Otovent manufacturer's limit. 


\section{Funding}

This study was carried out by KM as an undergraduate research project. A small amount of funding from the University of Oxford Medical School was attached to this project and used to buy the balloons.

\section{Ethical approval}

Ethical approval for the pilot clinical study was obtained from the Central University Research Ethics Committee (CUREC), Oxford (R46496/RE001).

\section{Provenance}

Freely submitted; externally peer reviewed.

\section{Acknowledgements}

The authors would like to thank all the participants in the pilot clinical study for taking part. They would would also like to thank Jason Oke for advice on the use of mixed-effect models.

\section{Competing interests}

SF and KM have no competing interests. RP has received grants from the UK NIHR, Cancer Research UK, BHF, NIHR School of Primary Care Research and support from the NIHR Oxford Biomedical Research Centre and the NIHR Community Healthcare MedTech and In Vitro Diagnostics Co-operative. PG receives grants from the National Health \& Medical Research Council.

\section{References}

1. Rosenfeld RM, Shin JJ, Schwartz SR, et al. Clinical practice guideline: otitis media with effusion (update). Otolaryngol Head Neck Surg 2016; 154(1 Suppl): S1-S41. DOI: https://doi.org/10.1177/0194599815623467

2. Venekamp RP, Javed $F$, van Dongen $T M$, et al. Interventions for children with ear discharge occurring at least two weeks following grommet (ventilation tube) insertion. Cochrane Database Syst Rev 2016; 11(11): CD011684. DOI: https://doi.org/10.1002/14651858.CD011684.pub2

3. Butler CC, van Der Voort JH. Steroids for otitis media with effusion: a systematic review. Arch Pediatr Adolesc Med 2001; 155(6): 641-647. DOI: https://doi.org/10.1001/archpedi.155.6.641

4. Zielhuis GA, Rach GH, Van den Broek P. The occurrence of otitis media with effusion in Dutch pre-school children. Clin Otolaryngol Allied Sci 1990; 15(2): 147-153. DOI: https://doi.org/10.1111/j.1365-2273.1990.tb00448.x

5. Fiellau-Nikolajsen M. Tympanometry and secretory otitis media. Observations on diagnosis, epidemiology, treatment, and prevention in prospective cohort studies of three-year-old children. Acta Otolaryngol Suppl 1983; 394: 1-73.

6. Williamson I. Otitis media with effusion in children. BMJ Clin Evid 2011; 2011: 0502.

7. Lous J, De Melker RA. Secretory otitis media in schoolchildren. Is screening for secretory otitis media advisable? Dan Med Bull 1995; 42(1): 79-80. DOI: https://doi.org/10.3109/13814789509160768

8. Khanna R, Lakhanpaul M, Bull PD, ., Guideline Development Group Surgical management of otitis media with effusion in children: summary of NICE guidance. Clin Otolaryngol 2008; 33(6): 600-605. DOI: https://doi.org/10. $1111 / \mathrm{j} .1749-4486.2008 .01844 . x$

9. Medical Research Council Multicentre Otitis Media Study Group. Surgery for persistent otitis media with effusion: generalizability of results from the UK trial (TARGET) trial of alternative regimens in glue ear treatment. Clin Otolaryngol Allied Sci 2001; 26(5): 417-424. DOI: https://doi.org/10.1046/j.1365-2273.2001.00495.x

10. Paradise JL, Feldman HM, Campbell TF, et al. Effect of early or delayed insertion of tympanostomy tubes for persistent otitis media on developmental outcomes at the age of three years. N Engl J Med 2001; 344(16): 1179-1187. DOI: https://doi.org/10.1056/NEJM200104193441601

11. Griffin G, Flynn CA. Antihistamines and/or decongestants for otitis media with effusion (OME) in children. Cochrane Database Syst Rev 2001; 2011(9): CD003423. DOI: https://doi.org/10.1002/14651858.CD003423.pub3

12. Venekamp RP, Burton MJ, van Dongen TMA, et al. Antibiotics for otitis media with effusion in children. Cochrane Database Syst Rev 2016(6): CD009163. DOI: https://doi.org/10.1002/14651858.CD009163.pub3

13. Williams RL, Chalmers TC, Stange KC, et al. Use of antibiotics in preventing recurrent acute otitis media and in treating otitis media with effusion. A meta-analytic attempt to resolve the brouhaha. JAMA 1993; 270(11): 1344-1351.

14. Cantekin El, McGuire TW. Antibiotics are not effective for otitis media with effusion: reanalysis of meta-analyses. Otorhinolaryngol Nova 1998; 8(5): 214-222. DOI: https://doi.org/10.1159/000027879

15. Rosenfeld RM, Mandel EM, Bluestone CD. Systemic steroids for otitis media with effusion in children. Arch Otolaryngol Head Neck Surg 1991; 117(9): 984-989. DOI: https://doi.org/10.1001/archotol.1991.01870210056008

16. Simpson SA, Lewis R, van der Voort J, Butler CC. Oral or topical nasal steroids for hearing loss associated with otitis media with effusion in children. Cochrane Database Syst Rev 2011(5): CD001935. DOI: https://doi.org/10. 1002/14651858.CD001935.pub3 
17. Perera R, Glasziou PP, Heneghan CJ, et al. Autoinflation for hearing loss associated with otitis media with effusion. Cochrane Database Syst Rev 2013(5): CD006285. DOI: https://doi.org/10.1002/14651858.CD006285.pub2

18. Del Mar C, Hoffmann T. Autoinflation: an effective nondrug intervention for glue ear. CMAJ 2015; 187(13): 949-950. DOI: https://doi.org/10.1503/cmaj.150527

19. Stangerup SE. Autoinflation: historical highlights and clinical implications. Ear Nose Throat J 1998; 77(9): 737-742. DOI: https://doi.org/10.1177/014556139807700910

20. Appelboam A, Reuben A, Mann C, et al. Postural modification to the standard Valsalva manoeuvre for emergency treatment of supraventricular tachycardias (REVERT): a randomised controlled trial. Lancet 2015; 386(10005): 1747-1753. DOI: https://doi.org/10.1016/S0140-6736(15)61485-4

21. Lu K, Clark JW, Ghorbel FH, et al. A human cardiopulmonary system model applied to the analysis of the Valsalva maneuver. Am J Physiol Heart Circ Physiol 2001; 281(6): H2661-H2679. DOI: https://doi.org/10.1152/ajpheart. 2001.281.6.H2661

22. Schlegel TT, Benavides EW, Barker DC, et al. Cardiovascular and Valsalva responses during parabolic flight. J Appl Physiol 1998; 85(5): 1957-1965. DOI: https://doi.org/10.1152/jappl.1998.85.5.1957

23. Blanshard JD, Maw AR, Bawden R. Conservative treatment of otitis media with effusion by autoinflation of the middle ear. Clin Otolaryngol Allied Sci 1993; 18(3): 188-192. DOI: https://doi.org/10.1111/j.1365-2273.1993. tb00827.x

24. Hopper J, Gould C. Applied cardiovascular physiology. Anaesthesia \& Intensive Care Medicine 2016; 17(5): 258-261. DOI: https://doi.org/10.1016/j.mpaic.2016.02.009

25. Stangerup SE, Sederberg-Olsen J, Balle V. Autoinflation as a treatment of secretory otitis media. A randomized controlled study. Arch Otolaryngol Head Neck Surg 1992; 118(2): 149-152. DOI: https://doi.org/10.1001/archotol. 1992.01880020041013

26. A'Court $C$, Stevens $R$, Sanders $S$, et al. Type and accuracy of sphygmomanometers in primary care: a crosssectional observational study. Br J Gen Pract 2011; 61(590): e598-e603. DOI: https://doi.org/10.3399/ bjgp11X593884

27. Brooker DS, McNeice A. Autoinflation in the treatment of glue ear in children. Clin Otolaryngol Allied Sci 1992; 17(4): 289-290. DOI: https://doi.org/10.1111/j.1365-2273.1992.tb00997.x

28. Williamson I, Vennik J, Harnden A, et al. Effect of nasal balloon autoinflation in children with otitis media with effusion in primary care: an open randomized controlled trial. CMAJ 2015; 187(13): 961-969. DOI: https://doi.org/ 10.1503/cmaj.141608 